\title{
Determinación del estado tensional de un invernadero tipo capilla en áreas de clima tropical
}

\author{
José Félix Morales Leslie ${ }^{1}$, Amaury Santiago Santana Reyes \\ Universidad de Granma, Bayamo, Cuba.
}

(Recibido: Febrero, 2016. Aceptado para publicación: Julio, 2016)

\begin{abstract}
Resumen:
El presente trabajo analiza la estructura de un invernadero tipo capilla, el que a través de un modelo simplificado es sometido a vientos tropicales de $120 \mathrm{~km}$, determinándose el estado tensional que presenta al actuar cargas de vientos en dos direcciones (lateral-frontal). En la investigación se muestran las tensiones Von Misses asociadas a los esfuerzos que provocan las cargas de vientos sobre cada elemento, comparándose los resultados con el límite elástico del material empleado, además se muestran los desplazamientos que ocurren en los nodos producto de la acción de las cargas, lo anterior se logra mediante el uso del programa RAM Advanse versión 5.1 aplicándose dos combinaciones de cargas que consideran el peso propio y las cargas de viento con sus respectivos coeficientes de mayoración, finalmente se presentan las conclusiones derivadas del análisis de los resultados obtenidos.
\end{abstract}

Palabras Claves: Invernadero; Carga de viento; Tensiones.

\begin{abstract}
:
The present work has to do with a chapel type greenhouse's structure analysis; which through a simplified model is subdued to $120 \mathrm{~km}$ 's tropical winds, determining the tensional situation that it shows when performing on loads of winds in two directions (lateral-frontal). In the investigation the Von Misses tensions are evidenced associated to the labors that cause the wind loads on each element, comparing the results with the elastic limit of the used material, besides, displacements are also offered which occur in the knots due to action loads. This is obtained by using the RAM Advanse program, 5.1 version; applying two load combinations that consider the weight itself and the wind loads with their respective increasing coefficients. Finally, the conclusions derived from the analysis of the obtained results are expressed.
\end{abstract}

Keywords: Greenhouse; Wind load; Stress.

\section{Introducción}

La preocupación de muchos países es satisfacer las necesidades de alimentación humana, y precisamente eso ha traído como consecuencia el desarrollo y la implementación de sistemas de producción, con los cuales el hombre produce sus alimentos. Una de las técnicas que se utiliza actualmente en la agricultura es el establecimiento de cultivos en ambientes protegidos o controlados. Este sistema es superior al método tradicional a cielo abierto, porque utiliza una cubierta que establece una barrera entre el medio externo y el cultivo, con el objetivo de proteger las plantas de la acción del viento, la lluvia, las plagas, las enfermedades, las plantas indeseables y los animales.

Durante los últimos años se ha incrementado el establecimiento de ambientes protegidos para la producción de plántulas en bandejas o contenedores, esta tendencia ha sido causada, entre otros factores, por la elevación de los costos de producción en los cultivos (uso de semillas u otros insumos importados de elevado precio) y por la necesidad de

\footnotetext{
${ }^{1}$ Autor para correspondencia. Email: jleslieme@udg.co.cu
} 
producir plantas sanas y de buena calidad para ser llevadas al campo, lo que se logra por la facilidad para la desinfección de los sustratos utilizados y por la separación del suelo como fuente potencial del inóculo (Ramírez, 2007).

No obstante, estudios realizados en países tropicales han demostrado que la adopción y desarrollo de esta tecnología ha presentado algunas limitantes. En primer lugar la mayoría de los productores carecen del conocimiento sobre manejo del cultivo y comportamiento de las plagas y enfermedades en el sistema, lo que cobra mayor relevancia si se considera que el conocimiento es la base fundamental para la innovación que nos puede llevar a una mejora de la productividad, rentabilidad y sostenibilidad. En segundo lugar los diseños de las estructuras no se han adaptado a las condiciones climáticas tropicales y socioeconómicas de los productores (Berdegué et al., 2007).

En Cuba desde el año 2006 se ha venido desarrollando de manera extensiva el uso de invernaderos, fundamentalmente del tipo "Túnel", con el objetivo de producir hortalizas fuera de estación, obteniéndose resultados favorables. En la isla se localizan 157.2 hectáreas donde se fomenta ese tipo de agricultura, típica de países desarrollados, distribuidas en dos mil 167 casas o instalaciones, (Marín, 2007).

Sin embargo, Cuba presenta los mismos problemas que la mayoría de los países tropicales donde se ha implementado esta tecnología, por lo que los rendimientos alcanzados comparados con los costos de fabricación, distan de las ganancias que realmente puede ofrecer su uso. Por todo lo anteriormente expuesto, se hace necesario introducir un nuevo modelo de casa que responda positivamente a las exigencias climáticas de la zona, más económica, que no necesite tecnología avanzada, que aumente la calidad y la cantidad de la producción y que cumpla con los parámetros de diseño establecidos para su construcción. Por lo anteriormente expuesto, se plantea como problema de esta investigación la necesidad de determinar el estado tensional de un invernadero tipo capilla con cubierta de 180 en condiciones de vientos tropicales.

\subsection{Objetivo General}

Determinar el estado tensional de un invernadero en condiciones de vientos tropicales.

\subsection{Objetivos Específicos}

- Obtener el modelo gráfico de la estructura objeto de estudio.

- Determinar el estado tensional de la estructura.

- Analizar los resultados derivados del estado tensional obtenido.

\section{Marco Teórico}

\subsection{Diseño de la estructura del invernadero}

En el diseño de estructuras se deberán tener en consideración varios aspectos, entre ellos el tipo de material a utilizar, el material de la cubierta, el cultivo patrón, las características del clima del lugar donde se va a instalar, entre otras. Lo anterior traerá consigo el éxito o no de la instalación.

El viento es una de los factores influyentes en el diseño de estructuras de invernaderos para climas tropicales, pues como es conocido existen fenómenos meteorológicos asociados al trópico, como las tormentas locales severas y los ciclones tropicales que 
pueden tener una influencia significativa en la estructura de estos. Por lo anterior es de vital importancia la determinación de las cargas de vientos y las de uso, que ha de soportar la estructura.

\subsection{Cargas actuantes}

Como se planteó en el epígrafe anterior, el viento es uno de los elementos a tener en consideración en el diseño de la estructura de un invernadero, por tal motivo se hace necesario un análisis del efecto que este pueda tener sobre la estructura del mismo, pues las características de estas dependerán del régimen eólico del emplazamiento, así como de las características geoclimáticas del lugar, por lo que se hace necesario realizar un estudio de las diferentes cargas a las que va a estar sometida la estructura entre las que se encuentran:

- Cargas permanentes: son aquellas que tanto durante la ejecución como durante la vida útil de la estructura actúan de forma constante. Se consideran cargas permanentes los pesos propios de todos los elementos que constituyen en este caso la estructura del invernadero.

- Carga de Uso: Las cargas de uso, de servicios o accidentales se diferencian de las cargas permanentes porque sus valores no son constantes, son temporales, teniendo sus duraciones y periodos de acción un carácter variable aleatorio.

- Cargas de Viento: Toda construcción, edificación o parte de ella se proyectará para resistir la acción del viento y existen normativas para el cálculo de la misma.

La Norma Cubana 285-2003 establece el método de cálculo para determinar la carga de viento que debe utilizarse en los cálculos estático y dinámico de edificios y obras de ingeniería, siendo la misma aplicable para estructura de invernaderos.

Considerando que estas cargas pueden actuar de manera simultánea en un momento dado sobre la estructura, se deben considerar en el estudio de su comportamiento combinaciones de cargas, que no es más que expresiones matemáticas que tienen en cuenta los diferentes tipos de cargas afectadas por los coeficientes de mayoración.

\subsection{Uso del método de los elementos finitos para la modelación de estructuras}

El método de los elementos finitos (MEF en español o FEM en inglés) es un método numérico de resolución de ecuaciones diferenciales. La solución obtenida por MEF es sólo aproximada y coincide con la solución exacta sólo en un número finito de puntos llamados nodos. En el resto de puntos que no son nodos, la solución aproximada se obtiene cuando se interpola a partir de los resultados obtenidos para los nodos, lo cual hace que la solución sea sólo aproximada (Such, 2009).

El MEF convierte un problema definido en términos de ecuaciones diferenciales en un problema en forma matricial que proporciona el resultado correcto para un número finito de puntos e interpola posteriormente la solución al resto del dominio, obteniéndose sólo una solución aproximada. El conjunto de puntos donde la solución es exacta se denomina conjunto de nodos. Dicho conjunto de nodos forma una red, denominada malla, formada por retículos. Cada uno de los retículos contenidos en dicha malla es un "elemento finito". El conjunto de nodos se obtiene de dividir o separar la estructura en elementos de forma variada, tales como superficies, volúmenes y barras (Jackson et al., 2005; Oñate, 2005; Zienkiewicz, 2007). 


\subsection{Tensión admisible de un elemento estructural}

Stiopin (1987), señala que para garantizar la resistencia de una viga es necesario que durante la flexión, las tensiones máximas de tracción y compresión en la sección peligrosa, es decir en la sección donde el momento flector tiene el valor máximo, no rebasen las correspondientes tensiones admisibles.

De lo anteriormente planteado se deduce la condición de resistencia siguiente:

$$
\sigma=\frac{M_{f l e c}}{W_{x}} \leq[\sigma]
$$

Donde:

$W_{x}$ : Momento axial de resistencia o módulo de resistencia a la flexión

$M_{\text {flec }}:$ Momento flector máximo

$[\sigma]$ : Tensión admisible

El momento axial de resistencia es una característica geométrica de la sección transversal de la viga, que determina su resistencia a la flexión. Se llama Módulo Axial de la Sección a la relación del momento de inercia respecto al eje dado a la distancia hasta el punto más alejado de la sección transversal. (Pisarenko et al, 1982).

$$
W_{x}=\frac{J_{x}}{y_{\max }}
$$

$J_{x}$ : Momento de inercia respecto al eje $\mathrm{x}$ de la sección

$y_{\max }$ : Distancia máxima hasta el eje $\mathrm{x}$ de la sección

Mediante el cálculo del módulo de resistencia a la flexión se determina el comportamiento resistivo de un elemento dado, sin embargo estas expresiones solo permiten resolver situaciones que responden a solicitaciones simples. Para determinar la resistencia de un elemento sometido a varias solicitaciones, las ecuaciones utilizadas en las solicitaciones simples no son suficientes, por lo que hay que acudir a las teorías o criterios de resistencia (Shames, 1975; Timoshenko, 1961; Shigley, 2008).

\subsection{Determinación de la carga de viento}

Para la determinación de la carga de viento según la Norma Cubana 285-2003 se realiza el cálculo mediante la siguiente fórmula:

$$
q=q_{10} \times C_{t} \times C_{s} \times C_{r} \times C_{h} \times C_{r a} \times C_{f}
$$

Donde:

q10: Presión básica del viento

$\mathrm{C}_{\mathrm{t}}$ : Coeficiente de recurrencia

Cs: Coeficiente de sitio 
Cr: Coeficiente de ráfaga

$\mathrm{C}_{\mathrm{h}}$ : Coeficiente de altura

$\mathrm{C}_{\text {ra: }}$ Coeficiente de reducción

$\mathrm{C}_{\mathrm{f}}$ : Coeficiente de forma

La presión básica (q10) puede ser calculada por la expresión:

$$
q_{10}=\frac{V^{2}{ }_{10}}{1,6 \times 10^{3}}
$$

Donde:

$V^{2}{ }_{10}$ : Velocidad característica del viento para un período básico de recurrencia a una altura de $10 \mathrm{~m}$ sobre el terreno, $(\mathrm{m} / \mathrm{s})$.

El coeficiente de recurrencia expresa la medida de ocurrencia de la presión básica para un periodo detiempo determinado. Las presiones de base señaladas para la recurrencia de 50 años, serán afectadas por el coeficiente de recurrencia $\left(\mathrm{C}_{t}\right)$ que se establecen en la Tabla 1, para ser utilizado en períodos de recurrencia de 5 a 100 años.

Tabla 1. Coeficiente de recurrencia

Fuente: NC: 285-2003

\begin{tabular}{|c|c|}
\hline Tiempo de recurrencia (años) & Coeficiente de recurrencia \\
\hline 100 & 1.15 \\
\hline 50 & 1.00 \\
\hline 25 & 0.90 \\
\hline 10 & 0.75 \\
\hline 5 & 0.70 \\
\hline
\end{tabular}

El coeficiente de topografía o de sitio (Cs) representa la influencia de caracteres topográficos definidos, sus valores se establen en la siguiente tabla:

Tabla 2. Coeficiente de Sitio

Fuente: NC: 285-2003

\begin{tabular}{|c|c|}
\hline Topografía & Coeficiente de Sitio \\
\hline Sitio normal & 1.00 \\
\hline Sitio expuesto & 1.10 \\
\hline
\end{tabular}

El coeficiente de ráfaga $\left(\mathrm{C}_{\mathrm{r}}\right)$ es empleado para tener en cuenta la naturaleza fluctuante de los vientos y su interacción con edificaciones y otras construcciones, teniendo en cuenta las características del terreno, los cuales se clasifican de la siguiente manera:

- Terreno Tipo A: Estos serán abiertos (llanuras, costas, orillas de laguna y represas, etc.).También es representativo de terrenos con obstáculos y edificaciones que no superen los $10 \mathrm{~m}$. Se considera costa una distancia hasta 500 metros a partir de la línea del mar. 
- Terreno Tipo B: Estos serán cubiertos con obstáculos y edificaciones que superen los $10 \mathrm{~m}$. (ciudades, zonas boscosas, etc.). Se considera representativo de este tipo de terreno, cuando las condiciones señaladas se mantengan en una distancia de 500 m o más, a partir de la edificación u obra.

- Terreno Tipo C: Estos serán los centros de grandes ciudades, en los que al menos el $50 \%$ de las edificaciones poseen una altura promedio mayor de $22 \mathrm{~m}$ o más. Esta condición debe prevalecer en la dirección analizada, en al menos una distancia de $800 \mathrm{~m}$ de construcciones ó 10 veces la altura del edificio u obra (el mayor de los dos).

Tabla 3. Coeficiente de ráfaga $\left(\mathrm{C}_{\mathrm{r}}\right)$

Fuente: NC: $285-2003$

\begin{tabular}{|c|c|c|c|c|c|c|c|c|c|c|c|}
\hline \multirow{2}{*}{$\begin{array}{c}\text { Tipo de } \\
\text { terreno }\end{array}$} & \multicolumn{10}{|c|}{ Altura ( m) } \\
\cline { 2 - 13 } & $<10$ & 10 & 20 & 30 & 40 & 50 & 60 & 70 & 80 & 90 & 100 \\
\hline $\mathrm{A}$ & 1.22 & 1.18 & 1.14 & 1.12 & 1.1 & 1.09 & 1.08 & 1.07 & 1.06 & 1.06 & 1.05 \\
\hline $\mathrm{B}$ & 1.46 & 1.36 & 1.28 & 1.24 & 1.21 & 1.18 & 1.17 & 1.15 & 1.14 & 1.13 & 1.12 \\
\hline $\mathrm{C}$ & 1.9 & 1.72 & 1.54 & 1.44 & 1.38 & 1.32 & 1.3 & 1.27 & 1.24 & 1.22 & 1.21 \\
\hline
\end{tabular}

La determinación del coeficiente de altura $\mathrm{C}_{\mathrm{h}}$ se hace mediante la siguiente tabla:

Tabla 4. Tipos de terrenos

Fuente: NC: $285-2003$

\begin{tabular}{|c|ccc|}
\hline \multirow{2}{*}{ Altura (m) } & \multicolumn{3}{|c|}{ Tipos de terrenos } \\
\cline { 2 - 4 } & $\mathbf{A}$ & $\mathbf{B}$ & $\mathbf{C}$ \\
\hline De 0 a 5m & 0.80 & 0.48 & 0.19 \\
\hline 10 & 1.00 & 0.65 & 0.30 \\
\hline 20 & 1.25 & 0.88 & 0.47 \\
\hline 30 & 1.42 & 1.05 & 0.62 \\
\hline 40 & 1.56 & 1.20 & 0.75 \\
\hline 50 & 1.67 & 1.32 & 0.87 \\
\hline 60 & 1.77 & 1.43 & 0.98 \\
\hline 70 & 1.86 & 1.53 & 1.08 \\
\hline 80 & 1.95 & 1.62 & 1.18 \\
\hline 90 & 2.02 & 1.71 & 1.28 \\
\hline 100 & 2.09 & 1.79 & 1.47 \\
\hline 125 & 2.24 & 1.97 & 1.59 \\
\hline 150 & 2.38 & 2.14 & 1.79 \\
\hline 175 & 2.50 & 2.29 & 1.98 \\
\hline 200 & 2.61 & 2.43 & 2.17 \\
\hline
\end{tabular}

El coeficiente de reducción $\mathrm{C}_{\mathrm{ra}}$ tiene un valor único para el edificio u obra correspondiente a la altura máxima del mismo.

Las presiones del viento actúan sobre los elementos constituyentes de una edificación u obra (paneles, vigas, columnas y otros) y son afectadas mediante un coeficiente de 
reducción en función de la mayor dimensión (horizontal o vertical) de la superficie o área expuesta al viento transmitiéndose la carga sobre el elemento considerado. La reducción solo se usará cuando la mayor dimensión señalada sea igual o mayor que $15 \mathrm{~m}$. (NC: 2852003).

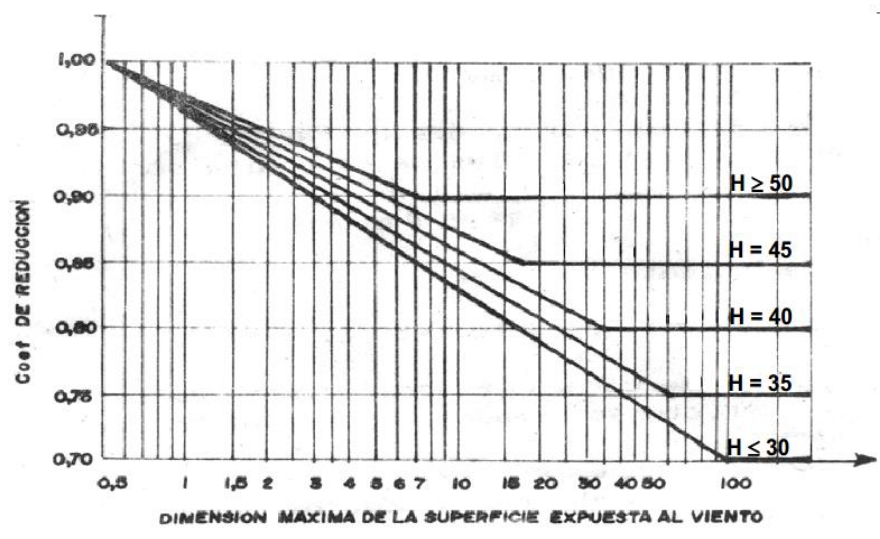

Figura 1. Selección del coeficiente de reducción Fuente: NC: $285-2003$

Las presiones básicas del viento están afectadas por el coeficiente de forma o aerodinámico $\mathrm{C}_{\mathrm{f}}$, el mismo considera la forma, los volúmenes y el ángulo de las superficies expuestas al viento, es decir el carácter aerodinámico de la estructura. Para determinar el coeficiente de forma se procederá seleccionándolo en la norma cubana NC: 285-2003 según la geometría del invernadero (ver Figura 2), a partir de los datos mostrados en las Tablas 4 y 5.

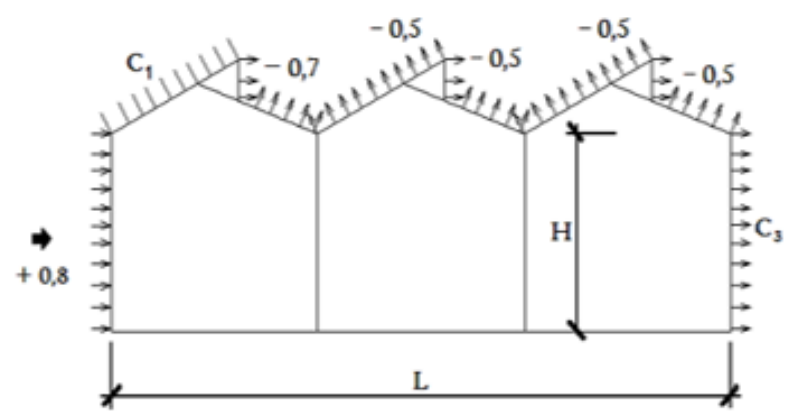

Figura 2. Selección de los coeficientes de forma Fuente: NC: $285-2003$

Tabla 5. Tabla para la determinación del coeficiente de forma $\mathrm{C}_{1}$ Fuente: NC: $285-2003$

\begin{tabular}{|c|c|c|c|c|c|}
\hline \multirow{4}{*}{} & \multirow{2}{*}{$\boldsymbol{\alpha}$} & \multicolumn{4}{|c|}{ H/L } \\
\cline { 3 - 6 } & & 0 & 0.5 & 1 & $\geq 2$ \\
\hline \multirow{4}{*}{$\mathrm{C}_{1} 1$} & 0 & 0 & -0.6 & -0.7 & -0.8 \\
\cline { 2 - 6 } & 20 & +0.2 & -0.4 & -0.7 & -0.8 \\
\cline { 2 - 6 } & 40 & +0.4 & +0.3 & -0.2 & -0.4 \\
\cline { 2 - 6 } & 60 & +0.8 & +0.8 & +0.8 & +0.8 \\
\hline \multicolumn{2}{|c|}{$\mathrm{C}_{2}$} & -0.4 & -0.4 & -0.5 & -0.8 \\
\hline
\end{tabular}


Tabla 6. Tabla para la determinación del coeficiente de forma $\mathrm{C}_{3}$

Fuente: NC: 285-2003

\begin{tabular}{|c|c|c|c|}
\hline \multirow{2}{*}{$\mathbf{B} / \mathbf{L}$} & \multicolumn{3}{|c|}{$\mathbf{H} / \mathbf{L}$} \\
\cline { 2 - 4 } & $\leq 0.5$ & 1 & $\geq 2$ \\
\hline$\leq 1$ & -0.4 & -0.5 & -0.6 \\
\hline$\geq 2$ & -0.5 & -0.6 & -0.6 \\
\hline
\end{tabular}

B: Largo del invernadero

L: Ancho del inverandero

H: Altura a canal

Todos los aspectos antes tratados son de gran importancia para el diseño y fabricación de invernaderos de acuerdo a las condiciones medioambientales del trópico, pues de no ser considerados pudieran traer como consecuencia alteraciones en los rendimientos productivos debido a dificultades con el clima interior dentro de la instalación, así como en la durabilidad de las mismas.

\section{Metodología y desarrollo}

\subsection{Características del invernadero objeto de estudio}

En el análisis de la estructura se tuvo en consideración los aspectos geométricos de la misma, el tipo de cubierta y que la estructura estaría sometida a velocidades de vientos de $120 \mathrm{~km} / \mathrm{h}$ valorando que en muchas zonas del trópico ocurren tormentas locales severas con velocidades de vientos superiores a $\operatorname{los} 100 \mathrm{~km} / \mathrm{h}$.

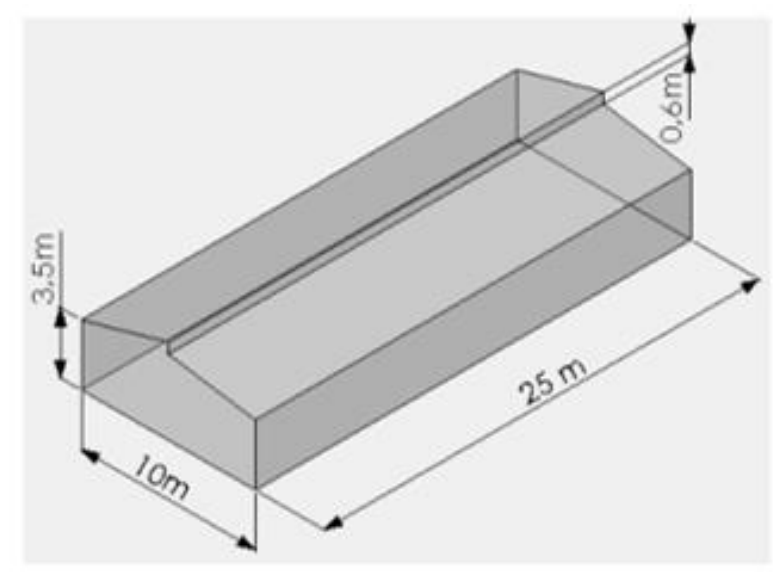

Figura 3. Dimensiones de invernadero objeto de estudio

Entre las características del invernadero se encuentran:

- Superficie cubierta: $250 \mathrm{~m}^{2}$

- Techo: Polietileno de alta densidad con un espesor de 200 micras.

- Ventana cenital de 0.6 x 25 m cubierta con malla mosquitera de 6 x 6 .

- Superficie ventana cenital: $87.5 \mathrm{~m}^{2}$ cada una.

- Superficies laterales hasta canal: $122.3 \mathrm{~m}^{2}$, cubierta con malla mosquitera 6 x 6 .

- Superficie frontal y trasera: $40.3 \mathrm{~m}^{2}$.

- B: $25 \mathrm{~m}$ 
- L: $10 \mathrm{~m}$

- $\mathrm{H}: 3.5 \mathrm{~m}$

Tabla 7. Listado de componentes

\begin{tabular}{|c|c|c|c|}
\hline Pos & Denominación & Material & Descripción \\
\hline 1 & Soporte de techo & A-36 & Tubo $60 \times 2$ \\
\hline 2 & Refuerzo Horizontal & A-36 & Tubo $60 \times 2$ \\
\hline 3 & Lateral Intermedio & A-36 & Tubo $60 \times 2$ \\
\hline 4 & Perfil M & A-36 & \\
\hline 5 & Lateral Extremo & A-36 & Tubo $60 \times 2$ \\
\hline 6 & Pilar Frontal & A-36 & Tubo $60 \times 2$ \\
\hline 7 & Pilar Central & A-36 & Tubo $60 \times 2$ \\
\hline 8 & Soporte de techo extremo & A-36 & Tubo $60 \times 2$ \\
\hline 9 & Pilar Central Horizontal & A-36 & Tubo $60 \times 2$ \\
\hline 10 & Pilar Frontal 1 & A-36 & Tubo $60 \times 2$ \\
\hline 11 & Lateral Extremo 1 & A-36 & Tubo $60 \times 2$ \\
\hline 12 & Lateral Intermedio Derecho & A-36 & Tubo $60 \times 2$ \\
\hline 13 & Soporte de techo 2 & A-36 & Tubo $60 \times 2$ \\
\hline 14 & Soporte de techo 3 & A-36 & Tubo $60 \times 2$ \\
\hline 15 & Pilar Central Superior & A-36 & Tubo $60 \times 2$ \\
\hline
\end{tabular}

A continuación se muestran los diferentes elementos que integran la estructura del invernadero objeto de estudio.

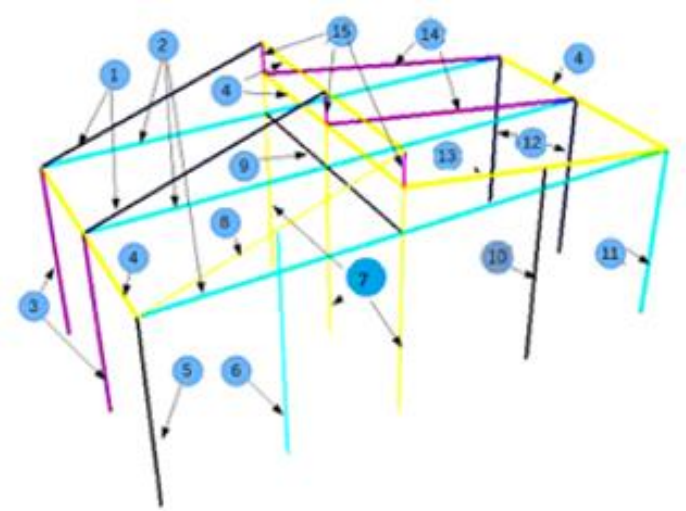

Figura 4. Modelo estructural simplificado

\subsection{Cálculo de cargas de viento lateral}

La carga de viento se determinó por la expresión siguiente, la que fue tomada de la Norma Cubana NC: 285-2003.

$$
\begin{gathered}
q_{L}=q_{10} \times C_{t} \times C_{s} \times C_{h} \times C_{r} \times C_{r a} \times C_{f}[\mathrm{kN} / \mathrm{m}] \\
q_{10}=\frac{V_{10}{ }^{2}}{1,6 \times 10^{3}}[\mathrm{kN} / \mathrm{m}]
\end{gathered}
$$

Coeficiente de recurrencia $\left(\mathrm{C}_{\mathrm{t}}\right)$ - Para la estructura analizada, se tomó un periodo de recurrencia de 50 años al cual corresponde un coeficiente $\mathrm{Ct}=1.00$. 
Coeficiente de topografía o de sitio $\left(\mathrm{C}_{\mathrm{S}}\right)$ - Para la estructura analizada corresponde un coeficiente $\mathrm{Cs}_{\mathrm{s}}=1.00$ por ser un sitio normal.

Coeficiente de altura $\left(C_{h}\right)$ - Para la estructura analizada, el coeficiente de altura $C_{h}=0.8$ por estar ubicada en un terreno tipo A y tener una altura de $4.5 \mathrm{~m}$.

Coeficiente de reducción $\left(\mathrm{C}_{\mathrm{ra}}\right)$ - El coeficiente de reducción utilizado $\mathrm{C}_{\mathrm{ra}}=0.74$, ya que la mayor dimensión corresponde al largo total del invernadero $(25 \mathrm{~m})$ y su altura $(\mathrm{H})$ es menor que $30 \mathrm{~m}$.

Coeficiente de forma $\left(\mathrm{C}_{\mathrm{f}}\right)$ - La determinación del coeficiente de forma se realizó atendiendo a lo planteado en la NC: 285-2003, seleccionándose la geometría que más se adapta al modelo objeto de estudio.

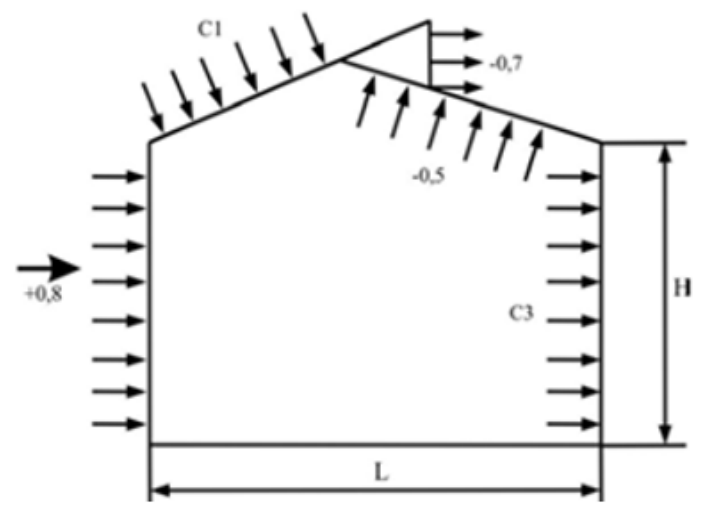

Figura 5. Esquema para la determinación de los coeficientes de forma Fuente NC: $285-2003$

Seguidamente se procedió a determinar las cargas de viento para cada uno de los componentes, basándose en un modelo simplificado; el conocimiento de la distancia entre cada pórtico de la estructura y la posición entre los componentes analizados es importante para efectuar el cálculo de las cargas que tributan a cada elemento, por lo que se hizo necesario en la concepción del modelo especificar con exactitud las coordenas que definen la ubicación de los pórticos según se muestra en la Figura 6.

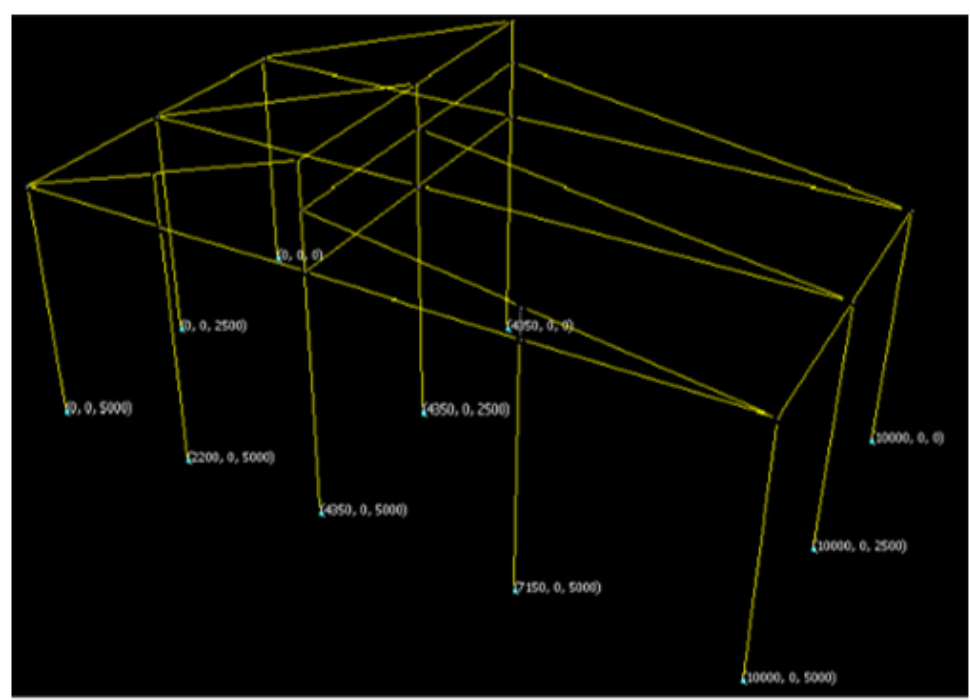

Figura 6. Ubicación de los pórticos 
Se calculó la carga $q_{L i}$ que actúa sobre los pilares laterales intermedios, dicha carga será afectada en un $32.8 \%$, pues la malla que cubre estos laterales tiene un $67.2 \%$ de ventilación.

$$
q_{L i}=q_{L} \times 2.5 m \quad[\mathrm{kN} / \mathrm{m}]
$$

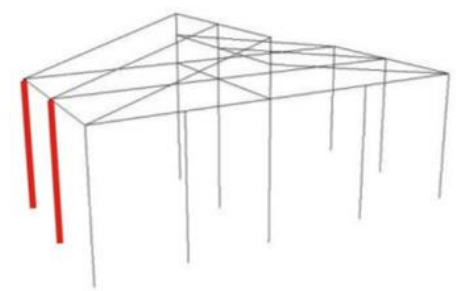

Figura 7. Pilares laterales intermedios

La carga en los pilares externos se calculó mediante la ecuación siguiente:

$$
q_{\text {lExterno }}=q_{L} \times 1,25 m *[\mathrm{kN} / \mathrm{m}]
$$

La carga de viento que actúa sobre los perfiles M se calculó utilizando la ecuación que se muestra a continuación:

$$
q_{L}=q_{L} \times 1.75 m *[\mathrm{kN} / \mathrm{m}]
$$

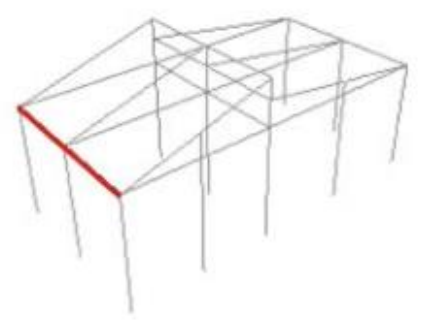

\section{Figura 8. Perfil M}

Se utilizó la siguiente ecuación para el cálculo de la carga de viento para los soportes de techo intermedios:

$$
\begin{gathered}
q_{S}=q_{10} \times C_{t} \times C_{S} \times C_{h} \times C_{r} \times C_{r a} \times C_{f}[\mathrm{kN} / \mathrm{m}] \\
q_{\text {stechoint }}=q_{S} \times 2.5 \mathrm{~m} *[\mathrm{kN} / \mathrm{m}]
\end{gathered}
$$

El símbolo * que se muestra indica la distancia a la que actúa la carga que tributa al elemento que se estudia, si tenemos en consideración que la distancia a la que están los postes, ubicados a ambos lados del pilar que se analiza es $2,5 \mathrm{~m}$ y que la carga de viento que actúa sobre la malla se divide entre los elementos, esta distancia es $1.25 \mathrm{~m}$ del soporte que le antecede y $1.25 \mathrm{~m}$ del que le sucede. Es por eso que la suma de las dos cargas que tributan al elemento que se analiza es $2.5 \mathrm{~m}$, es importante recordar que el modelo está simplificado y es por eso que solo se analizan tres pórticos. Este mismo análisis se realizó con el resto de los elementos.

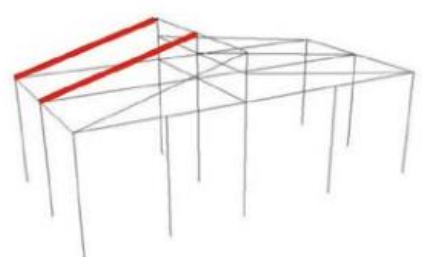

Figura 9. Soportes de techo intermedios 1 
La carga de viento para el soporte de techo extremo se calculó por la ecuación:

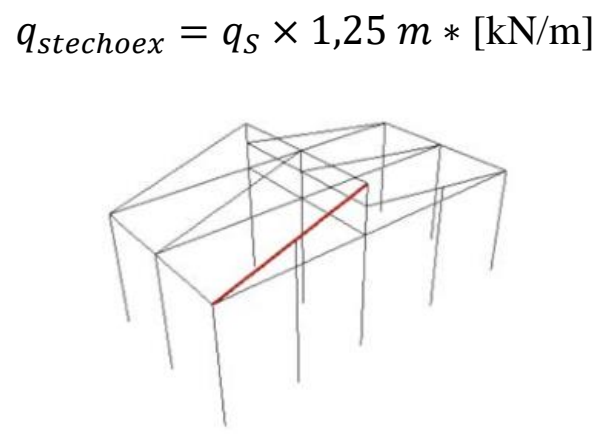

Figura 10. Soporte de techo extremo

Para el cálculo de las cargas de viento que actúan sobre los pilares centrales superiores se consideró que la carga global $q$, que inicialmente se calculó solo pasaba por la malla que cubre el lateral en un 67.2\%, de ahí que esa es la carga global que acciona sobre los elementos estructurales interiores.

$$
\begin{gathered}
q_{i}=q \times 0.672[\mathrm{kN} / \mathrm{m}] \\
q_{p c s}=q_{i} \times C_{f} \times a_{t u b o}[\mathrm{kN} / \mathrm{m}]
\end{gathered}
$$

$a_{t u b o}$ : Ancho del tubo de los pilares centrales superiores

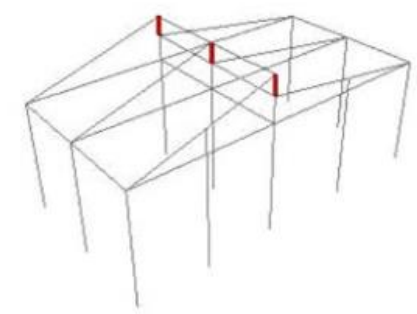

Figura 11. Pilares centrales superiores

Para el cálculo de la carga de viento que actúa sobre los soportes de techo 2 se utilizó la ecuación:

$$
q_{\text {sTecho } 2}=q_{i} \times C_{f} \times a_{\text {tubo }}[\mathrm{kN} / \mathrm{m}]
$$

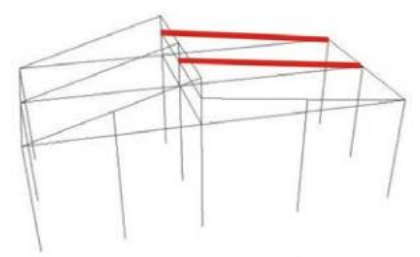

Figura 12. Soportes de techo 2

El cálculo de la carga de viento que actúa sobre los pilares laterales intermedio derechos se realizó por la ecuación siguiente:

$$
q_{\text {Lintermedio } D}=q_{i} \times C_{f} \times a_{\text {tubo }}[\mathrm{kN} / \mathrm{m}]
$$

El coeficiente de forma $\mathrm{C}_{\mathrm{f}}$ que se introduce en la expresión se selecciona de la Tabla 5. 


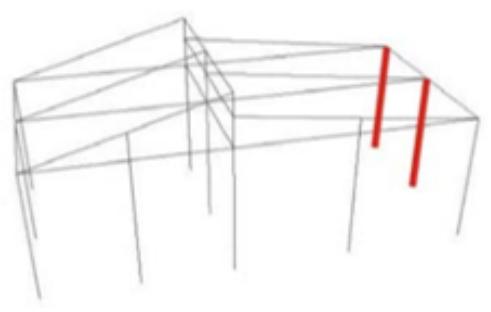

Figura 13. Pilares laterales intermedio derechos

Se calculó la carga de viento que actúa sobre los pilares centrales de la siguiente forma:

$$
q_{p c}=q_{i} \times a_{t u b o}[\mathrm{kN} / \mathrm{m}]
$$

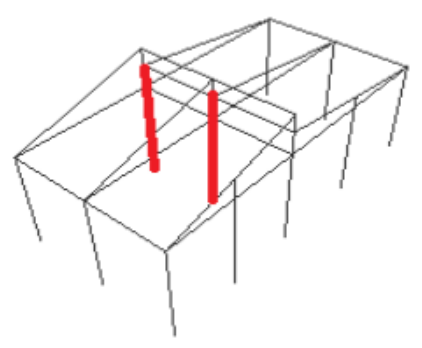

Figura 14. Pilares centrales

\subsection{Determinación de las cargas de vientos frontales}

La dirección del viento a analizar tiene una incidencia en las cargas que actúan sobre la estructura. La determinación de la carga de viento que actúa sobre el pilar lateral extremo se calcula de la siguiente forma:

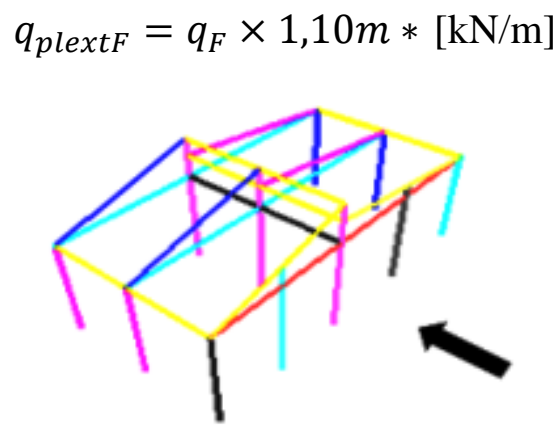

Figura 15. Dirección del viento a analizar

La magnitud 1,10m es la distancia a la que tributa la carga que actúa sobre el elemento analizado, que no es más que la mitad de la distancia que existe entre el elemento que se analiza y el elemento que le sucede, que en este caso es $2.2 \mathrm{~m}$.

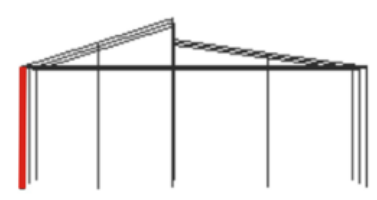

Figura 16. Pilar lateral extremo

Se calculó la carga de viento para el pilar frontal mediante la siguiente ecuación: 


$$
q_{\text {pronF }}=q_{F} \times 2,17 m *[\mathrm{kN} / \mathrm{m}]
$$

La magnitud 2,17m es la distancia a la que actúa la carga que tributa al pilar frontal, teniendo en cuenta la distancia que existe entre los pilares (pilar lateral extremo y el pilar central), que están a ambos lados del elemento que se analiza. La distancia existente entre el pilar lateral extremo y el pilar frontal es de $2.20 \mathrm{~m}$ y entre este último y el pilar central $2,15 \mathrm{~m}$, por tanto la carga actuante $\left(q_{p f r o n}\right)$, no es más que el múltiplo de la carga frontal $\left(q_{F}\right)$ y la suma de mitad de las distancias antes mencionadas.

$$
\begin{gathered}
q_{\text {pfronF }}=q_{F} \times 1,1 m+q_{F} \times 1,07 m[\mathrm{kN} / \mathrm{m}] \\
q_{\text {pfronF }}=q_{F} \times(1,1+1,07) m[\mathrm{kN} / \mathrm{m}]
\end{gathered}
$$

Figura 17. Pilar Frontal

La carga de viento que actúa sobre el pilar central se obtiene de la ecuación:

$$
q_{\text {pcenF }}=q_{F} \times 2,47 m
$$

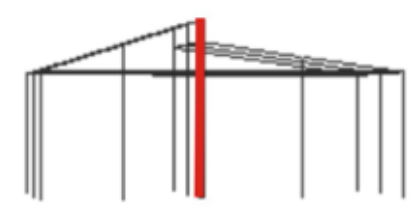

Figura 18. Pilar central

Se calculó la carga de viento que actúa sobre el pilar frontal 1 a través de la ecuación:

$$
q_{\text {pront } 1 F}=q_{F} \times 2,82 m
$$

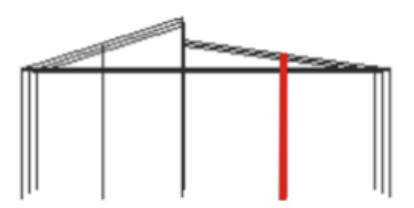

Figura19. Pilar frontal 1

La carga de viento que actúa sobre el pilar lateral extremo extremo se calculó mediante de la ecuación:

$$
q_{\text {lextreF }}=q_{F} \times 1,75 m
$$




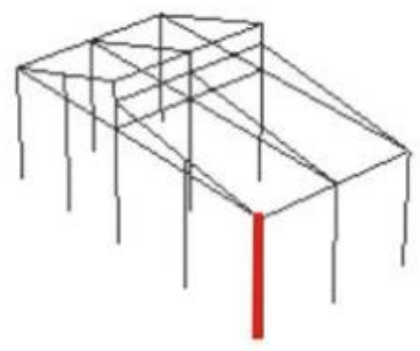

Figura 20. Lateral extremo

El cálculo de la carga de viento que actúa sobre el refuerzo horizontal se realizó por la ecuación:

$$
\begin{array}{||l|l||}
q_{\text {refuerHF }}=q_{F} \times 1,75 m \\
\end{array}
$$

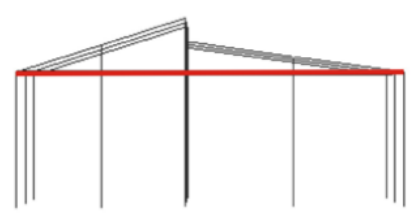

Figura 21. Refuerzo horizontal

\subsection{Implementación de las combinaciones de cargas que actúan sobre la estructura}

Las combinaciones de cargas que se establecieron atendiendo a los estados de carga a que está sometida la estructura fueron las siguientes:

$$
\begin{aligned}
& C C_{1}=1,1 p p+1,2 q v \\
& C C_{2}=1,1 p p+1,2 q f
\end{aligned}
$$

Siendo:

$C_{1}$ : Combinación de carga cuando el viento actúa por el lateral izquierdo

$C C_{2}$ : Combinación de carga cuando el viento actúa por el lateral derecho

$p p$ : peso propio del elemento

$q v$ : carga de viento por el lateral

$q f$ : carga de viento frontal

\subsection{Asignación de restricciones y cargas en el software}

Las restricciones consideradas fueron los empotramientos de los pilares. Según se muestra en la Figura 22. La declaración de éstas se realizó basada en las prestaciones que brinda el programa RAM Advanse versión 5.1.

Es importante aclarar que el modelo se simplificó para facilitar la corrida del programa. 


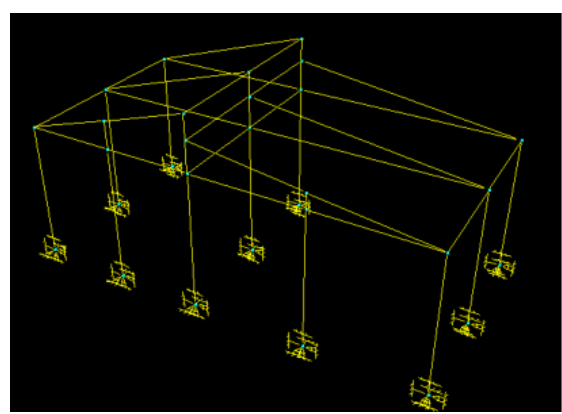

Figura 22. Declaración de las restricciones

En la Figura 23 se muestra la ubicación de las cargas debido a la acción lateral del viento. Colocadas las cargas se realizó una primera corrida atendiendo a la combinación de carga $\mathrm{CC}_{1}$ con el propósito de observar el comportamiento de la estructura a la acción del viento por el lateral izquierdo, obteniendo el estado tensional correspondiente.

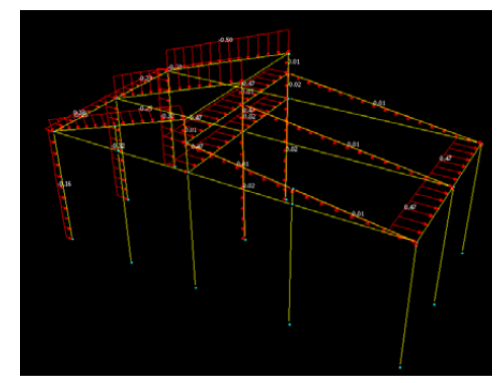

Figura 23. Localización de las cargas de vientos laterales

Las cargas frontales se muestran en la Figura 24; colocada estas cargas, se realizó una corrida del programa para observar el comportamiento de la estructura debido a la acción simultánea del peso propio y la carga de viento frontal, lo que corresponde a correr la combinación $\mathrm{CC}_{2}$.

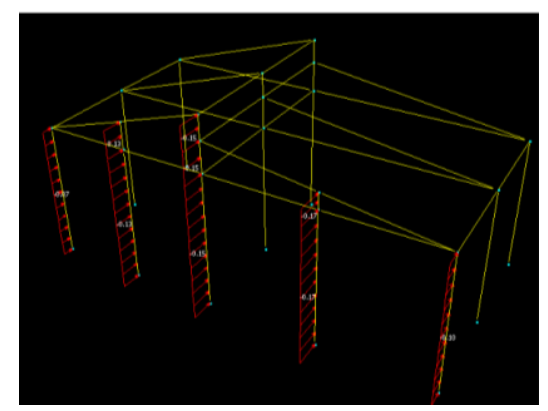

Figura 24. Localización de las cargas de vientos frontales

Es importante plantear que las cargas de uso no fueron consideradas para un análisis independiente, pues se tuvieron en cuenta en los coeficientes de mayoración del peso propio.

\section{Resultados del cálculo de las cargas de vientos que inciden sobre la estructura}

El cálculo de las cargas de viento tanto lateral como frontal arrojó los resultados que se muestran en las Tablas 7 y 8 . En la Tabla 7 se observa que el elemento más cargado es el "Soporte de techo 1", que podría ser debido a que la cubierta del mismo no es porosa, incidiendo en que la carga se transmita en su totalidad al elemento. 
Tabla 8. Resultado del cálculo de la carga de viento lateral

\begin{tabular}{|l|c|}
\hline \multicolumn{1}{|c|}{ Carga de viento lateral } & kN/m \\
\hline Presión Básica del viento & 0.69 \\
Carga de viento & 0.40 \\
Carga de viento por el lateral & 0.13 \\
Carga de viento sobre el pilar intermedio & 0.32 \\
Carga de viento sobre el pilar lateral extremo & 0.16 \\
Carga de viento sobre el perfil M & 0.23 \\
Soporte de techo 1 & $\mathbf{0 . 5 0}$ \\
Soporte de techo extremo & 0.25 \\
Pilar centrales superiores & 0.01 \\
Soporte de techo 2 & 0.01 \\
Soporte de techo 2 extremo & 0.01 \\
Pilar lateral intermedio derecho & 0.01 \\
Pilar centrales & 0.02 \\
\hline
\end{tabular}

En el caso de la carga de viento frontal se puede observar que la carga de viento más significativa se manifestó en el pilar frontal, lo cual está dado por el área efectiva expuesta al viento y la separación existente entre los pilares inmediatos.

Tabla 9. Resultado de la aplicación de la carga de viento frontal

\begin{tabular}{|l|c|}
\hline \multicolumn{1}{|c|}{ Carga de viento frontal } & $\mathbf{k N / m}$ \\
\hline Presión Básica del viento & 0.69 \\
Carga de viento & 0.20 \\
Carga de viento frontal & 0.06 \\
Carga de viento sobre el pilar lateral extremo & 0.07 \\
Carga de viento sobre el pilar frontal & 0.13 \\
Carga de viento sobre el pilar central & 0.15 \\
Carga de viento sobre el pilar frontal 1 & $\mathbf{0 . 1 7}$ \\
Carga de viento sobre el lateral extremo 1 & 0.08 \\
Carga de viento sobre el refuerzo horizontal & 0.10 \\
\hline
\end{tabular}

\subsection{Estados tensional debido a la acción de las carga de viento lateral}

Como resultado de la aplicación de la combinación de carga $C C_{1}$, se obtuvo el estado tensional o tensiones Von Misses mostrado en la Figura 25, las que se compararon con el límite de fluencia del material utilizado en los componentes de la estructura.

La máxima tensión a que está sometida la estructura es de $528,785.03 \mathrm{kN} / \mathrm{m}^{2}$ y el límite de fluencia del material de la estructura (A-36) es de $256,457.35 \mathrm{kN} / \mathrm{m}^{2}$, lo que demuestra que la estructura está sometida a tensiones que duplican el límite de fluencia de la misma, por lo que la estructura fallará al actuar vientos de $120 \mathrm{~km}$ en la dirección analizada, debiéndose analizar la posibilidad de utilizar elementos con mayor momento resistivo. 

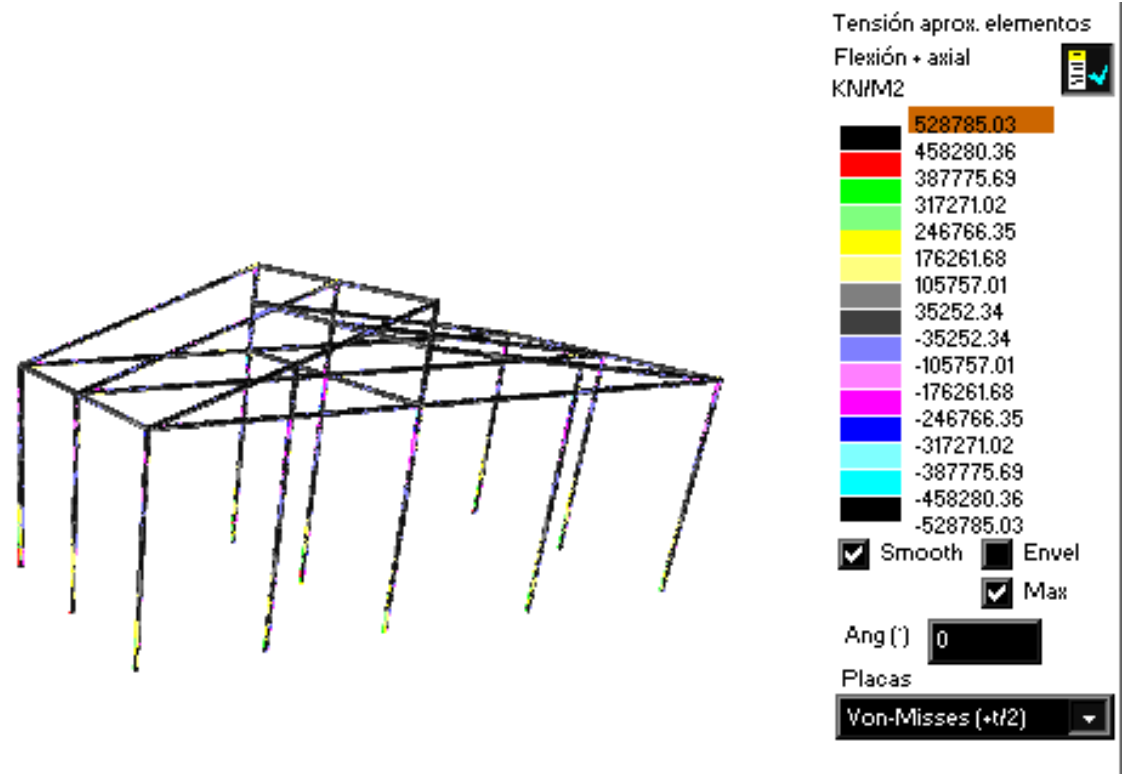

Figura 25.Tensiones debido a la acción de la carga de viento lateral

Los resultados de la aplicación de la combinación de carga $C C_{2}$ se muestran a través de la Figura 26, pudiéndose observar que la máxima tensión Von Misses es de 111,589.36 $\mathrm{kN} / \mathrm{m}^{2}$ la cual no supera el límite de fluencia del material, la estructura no falla bajo la acción de vientos en dirección frontal.

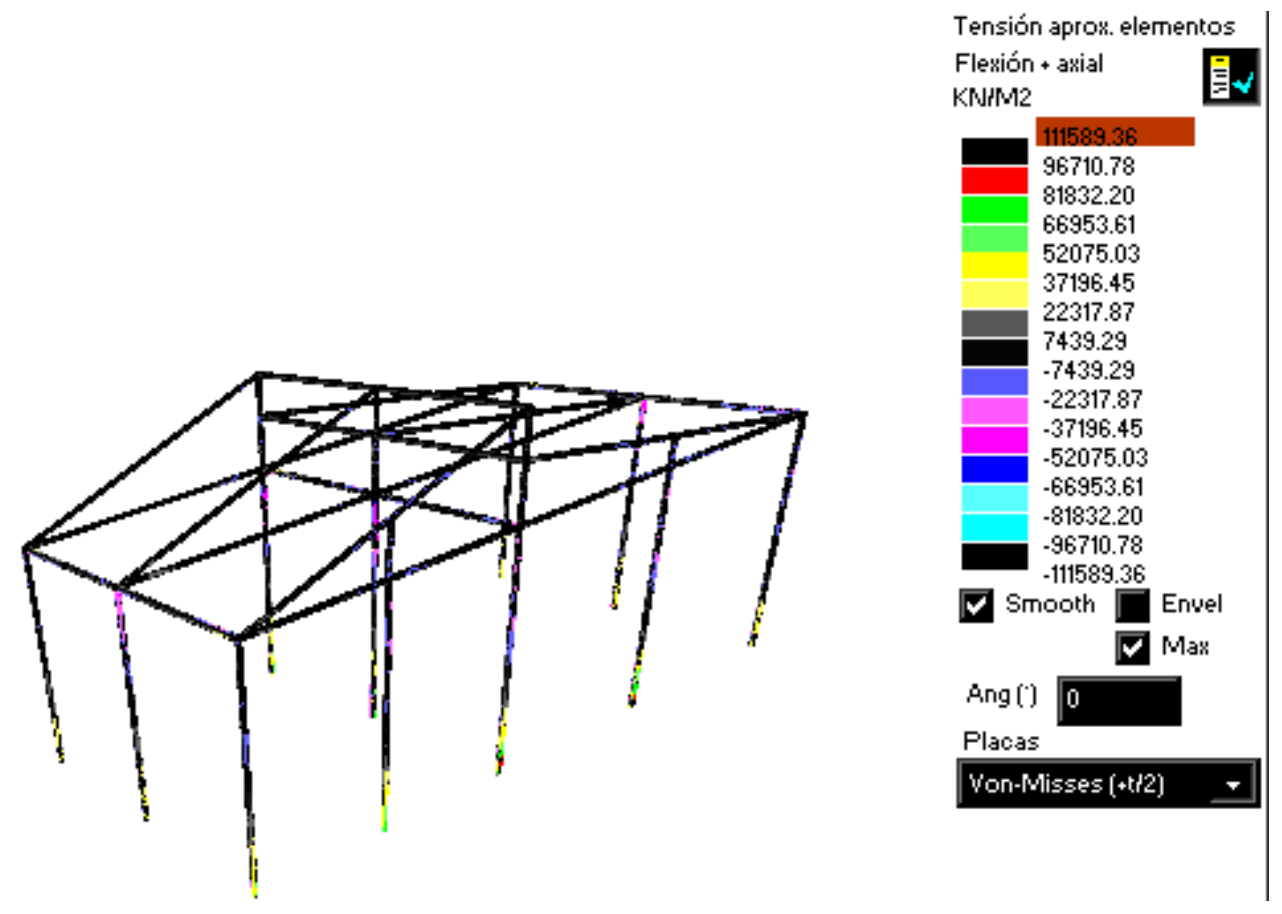

Figura 26. Tensiones debido a la acción de la carga de viento frontal

\section{Conclusiones}

1. El estado tensional de la estructura objeto de estudio demostró que el perfil redondo de 60 × $2 \mathrm{~mm}$ no es capaz de soportar los vientos de $120 \mathrm{~km} / \mathrm{h}$

2. El tipo de cubierta del invernadero influye de manera significativa en la acción de las cargas de viento. 
3. La distancia entre elementos es un factor a considerar para disminuir los efectos de las cargas de viento, pudiéndose tomar alternativas ingenieriles que permitan disminuir el peso de la estructura y el consiguiente costo.

\section{Bibliografía}

Berdegué, J., A. Ocampo, et al. (2007). Sistematización de experiencias locales de desarrollo rural. Guíametodológica, Fidamerica y Preval.

Jackson, L. (2005). Finite element study of the residual stress and deformation in hemispherical contacts, Transactions of the ASME.

Marín, L. (2007). Análisis estructural de invernadero MSC modelo tropical. Facultad de Ingeniería. Holguín, Universidad de Holguín "Oscar Lucero Moya” Centro de Estudios CAD- CAM. Tesis de Maestría.

NC: 285-2003 Carga de Viento. Métodos de Cálculo. http://www.eird.org/cd/acs/English/CodeEval/SpaSpeak/Wind/CUBAwce.pdf

Oñate, E. (2005). Cálculo de estructuras por el MEF. Análisis estático lineal. Barcelona, CIMNE.

Pisarenko, G. (1982). Manual de Resistencia de materiales. Moscú, Mir.

RAM Advansed 5.1 (2003). Manual instructivo de RAM Advanse.

Ramírez, H. (2007). La producción de plántulas de hortalizas en bandejas y ambientes protegidos. Curso de Producción de Hortalizas en Ambientes de Protegidos.

Shames, I. (1975). Introduction to Solid Mechanics. Prentice Hall Inc.

Shigley, J. and C. Mischke (2008). Mechanical engineering design. Mexico, The McGraw-Hil.

Stiopin, P. A. (1987). "Manual de Resistencia de Materiales”, p. 370.

Such, M., J. Jimenez, et al. (2009). An approach based on the catenary equation to deal with static analysis of three dimensional cable structures. In Press, CorrectedProof, Available online.

Timoshenko, P. and M. Gere, (1961). Theory of Elastic Stabilityll. New York, McGrawHill.

Zienkiewicz, O. and R. Taylor (2007). El método de los elementos finitos. Mecánica de sólidos. Barcelona, CIMNE.

\footnotetext{
"LA REVISTA INNOVARE NO SE HACE RESPONSABLE EN NINGÚN CASO DE LOS CONTENIDOS, DATOS, CONCLUSIONES U OPINIONES VERTIDAS EN LOS ARTÍCULOS PUBLICADOS, SIENDO ESTA RESPONSABILIDAD EXCLUSIVA DEL (DE LOS) AUTOR (AUTORES)"
} 